\title{
THE APPROACH TO WEB SERVICES COMPOSITION
}

\author{
Koval O., Globa L., Novogrudska R.
}

National Technical University of Ukraine «Kyiv Polytechnic Institute», Information Telecommunication Networks Department, Peremoga Ave. 37, 03056 Kyiv, Ukraine avkovalgm@gmail.com,lgloba@its.kpi.ua,rinan@ukr.net

\begin{abstract}
The paper presents an approach to the composition of web services based on their meta descriptions. The process of web services sequence formation is depicted. Such web services sequence describes execution of certain user's task. The method of user's tasks scenario formation is proposed that allows to define dynamically an ordered sequence of web services required to run a specific user's tasks. The scenario formation for real user's task «Calculation of the strength for the power components of magnetic systems» is represented, approving the applicability and efficiency of proposed approach.
\end{abstract}

Key words: web services, scenario, user's task, meta description, composition.

\section{$1 \quad$ Introduction}

Nowadays, a large number of web services are provided over the web from companies such as Google, Microsoft and Amazon. By utilizing these services, end user is expected to create value added services to fulfil the requirement. This process is commonly known as web services composition. An analysis of the literature related to the service composition focuses that, frequently performed tasks throughout the composition are the discovery and selection of the candidate web services which are participating to generate the composition plan. Although, there is a close relationship among discovery, selection and composition, researchers treat them individually and independently.

Web services usually are used for different processes performance. Such process can be:

- General user's tasks,

- Calculation and computational tasks,

- Business processes.

Moreover, different users want to achieve the increased value of satisfaction from the resultant composition solution. Thus, the execution of user's tasks is closely correlated with web services execution. User's task is the conceptual notion. For the execution of user's task, the sequence of web services must be executed (for the web services that correlate to this user's task). Web services composition into such sequence should be optimal to provide correct execution of user's tasks.

The main objective of the web services composition "on the fly" is the organization in the dynamics of such an ordered sequence of web services that allows to execute a specific end-user task. Thus, it is needed to develop the method of web services dynamic integration for a specific end-user task execution.

This paper describes a novel approach for web services composition given their meta descriptions that is based on the method of user's tasks scenario formation. 
The paper is structured as follows: Section 2 provides the background and analyses of related works on web services composition. Section 3 gives main notions and their formal description as well as characteristic features of web services. In this section the system of meta descriptions for web services and user's tasks is suggested, such system forms the basis of web services composition. Section 4 describes the method of user's tasks scenario formation. Section 5 contains the description of the proposed method application for the scenario formation that represent the execution of real complex computational user's task in the subject domain "Strength of materials". Section 6 concludes the work with a summary and outlook on future work.

\section{Backgrounds and related work}

Web Services Composition (WSC) is a method to connect different web services that are used for creating high-level business architecture by compiling of web services in order to provide functionalities that are not available during design [1]. Consequently, there is a possibility to develop a new functionality by simply reusing of components that are already available, but unable to complete a task successfully on their own.

Static and Dynamic WSC are two major types of web services composition. Dynamic web service composition occurs when user or software application queries for a web services at runtime while static web services composition is performed at the compile time. Dynamic web services composition is less trivial and requires much more work compared to static web services composition.

Various authors classify other WSC approaches. In [2], Static and Dynamic Web Services Composition approaches are grouped into a single approach. This and other possible approaches for composition are listed below:

- Static and Dynamic Composition ;

- Model Driven Service Composition;

- Declarative Service Composition;

- Automated and Manual Composition;

- Context-based Service Discovery and Composition.

The ability to select and compose heterogeneous web services over the web efficiently and effectively at runtime is an important step towards the development of the web services applications [3]. By utilizing web services, end user is able to create composite services to fulfil the requirement when single service unable to do it.

Most of the approaches related to the web services composition $[4,5,6,7]$ realized the fact that the prerequisite tasks to generate the composition solution are the service discovery and service selection of the candidate web services stored in the service repository.

Semantic web services $[8,9,10,11]$ provide an open, extensible, semantic framework for describing and publishing semantic content, improved interoperability, automated service composition, discovery and invocation, access to knowledge on the Internet [12].

$[13,14,15]$ describes discovery and composition issues of web services in the proposed extended SOA architecture. The concept of automated service discovery and composition process using Semantic Web services is presented.

In agent-based solutions for web services, composition agencies gather QoS data from agents, store, aggregate, and present it to agents [16]. Approach, which solves 
these problems, is introduced in [17]. Distinctive feature of this approach is utilization of SLA via WS-Agreement [18] during both workflow enactment and workflow analysis stages.

A composite service is a set of individual services that are effectively combined and reused in order to achieve a desired effect. Automatic service composition consists of four phases: Discovery, Selection, Planning, and Execution [19]. The first phase involves creating a plan, i.e., sequence of services in desired composition. The plan creation could be manual, semi-automatic, or automatic. The second phase embodies service discovery due to the plan. Planning and discovery are often combined into one step. After discovery of suitable services, the selection phase starts. It embodies a selection of the optimal composition from the available combinations of web services judging on nonfunctional properties like QoS properties. The final phase involves executing the services due to the plan. If some service is not available, another one takes its place. Concerning WS performance on engineering knowledge portals the most time-consuming is the phase of Discovery. As it is in need to choose from WS variety only those that are to be composed for complex engineering calculation performance.

In the case of solving the problem of web services composition aiming specific end-user's tasks execution it is in need to override these stages meaning. First it is needed to discover and select from web services set those web services that are used for described user's task execution. Then the user's task scenario is to be formed from this web services. Next the stage of execution is performed.

\section{The characteristic features of web services}

Fig. 1 describes the correlation between notions: user's task (UT), scenario and web service (WS). Each user's task stage is associated with certain web service that execute this stage. The scenario is an ordered sequence of this web services, scenario presents the conceptual process of user's task execution.

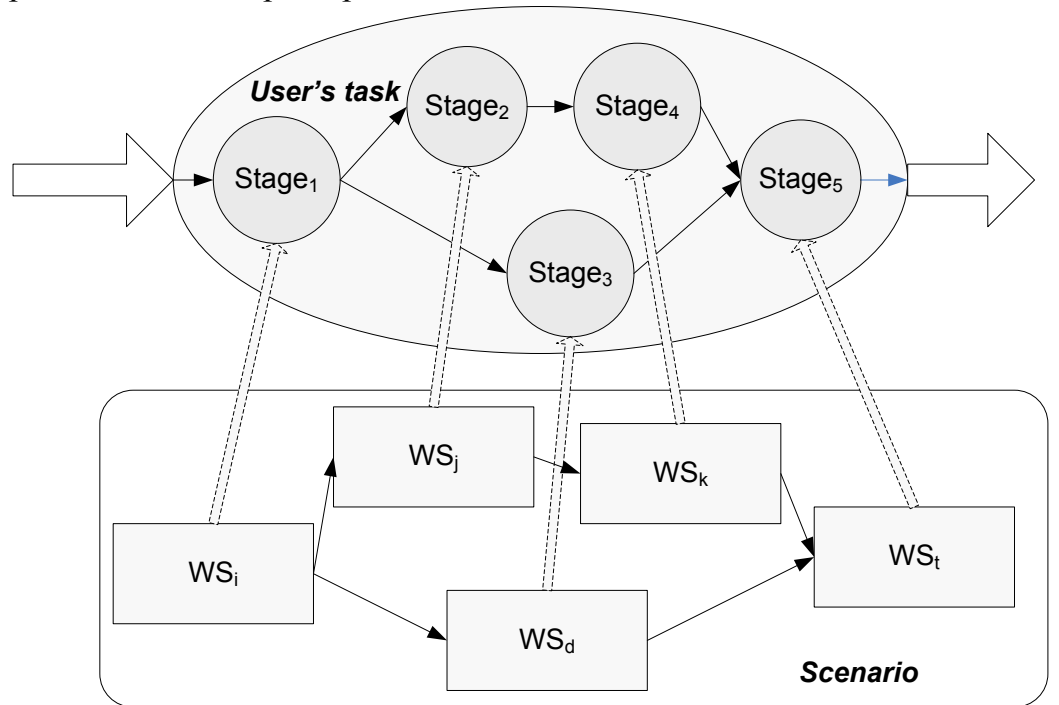

Fig. 1. Correlation between notions: scenario, user's task and web services 
Each of user's task has a specific set of attributes, describing its features and characteristics. As well, each WS can be described by some set of parameters. Such sets forms their meta descriptions. Specification of WS meta descriptions is based on Dublin Core standard and defines meta description as $M D=<A, D>$, where $A$ is meta description attribute (represent important characteristic or parameter of task or WS) and $D$ is data (represent the name of this parameter or characteristic for appropriate task or WS). Thus, each task and web service can be described by certain set of meta descriptions and each of such meta description has some meaning exactly for this task or WS. Such system of meta description allows to describe task or WS in terms of their parameters meanings for appropriate subject domain (that is not depicted by WS-CDL or WS-BPEL).

The structure of user's tasks meta descriptions and web services meta descriptions is similar. Thus, the dynamic generation of web services sequence (the scenario) that corresponds to the certain user's task can be based on the comparison of such web services meta descriptions and user's task meta descriptions.

All meta descriptions corresponds to two types:

1. General meta descriptions - describe different general characteristics of user's tasks and web services.

2. Specific meta descriptions - describe different parameters and characteristics of user's tasks and web services that depend on UTs and WSs subject domain (they reflect the specific of subject domain and may indicate the services necessary to perform a certain user's task).

Table 1 presents the suggested system of meta description for user's tasks and web services.

Table 1

\begin{tabular}{|c|c|}
\hline Meta descriptions title & Meta descriptions description \\
\hline \multicolumn{2}{|r|}{ Standard meta descriptions } \\
\hline Title & Title of UT or WS \\
\hline Identifier & $\begin{array}{l}\text { Unique ID number that is considered as UT or WS } \\
\text { number }\end{array}$ \\
\hline Subject domain & Subject domain of UT or WS \\
\hline Description & Text description of web service \\
\hline Data & $\begin{array}{l}\text { Data type, for the data that is used for UT or WS } \\
\text { execution }\end{array}$ \\
\hline Regulatory & $\begin{array}{l}\text { Title of the normative document (GOST, ISO, } \\
\text { Standard), guiding which user's task is executed }\end{array}$ \\
\hline Address & The reference to web service location \\
\hline \multicolumn{2}{|c|}{$\begin{array}{c}\text { Specific meta descriptions (as an example - specific meta descriptions for subject } \\
\text { domain "Strength of materials") }\end{array}$} \\
\hline Parameters & $\begin{array}{l}\text { Parameters that are used for engineering calculation } \\
\text { task (user's task) }\end{array}$ \\
\hline Loading & $\begin{array}{l}\text { Loading types that are used for engineering calculation } \\
\text { task (user's task) }\end{array}$ \\
\hline
\end{tabular}




\begin{tabular}{|l|l|}
\hline Method & $\begin{array}{l}\text { Method of calculation that is used within engineering } \\
\text { calculation task (user's task) execution }\end{array}$ \\
\hline Element & $\begin{array}{l}\text { Construction element that is used in engineering } \\
\text { calculation task (user's task) }\end{array}$ \\
\hline Measurement area & $\begin{array}{l}\text { The area of construction that is used in engineering } \\
\text { calculation task (user's task) }\end{array}$ \\
\hline
\end{tabular}

All web services are stored in independent storage, while meta descriptions and their values are stored in database tables (Fig. 2).

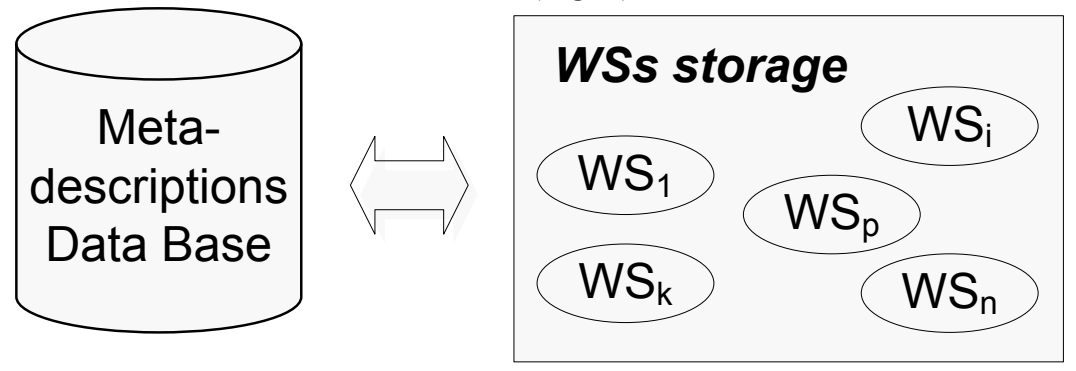

Fig. 2. Storage of WSs and meta descriptions

User's tasks set is described as:

$$
U T \ni U T_{i}, U T_{i}=\left\langle T_{i}, M D_{i p}\right\rangle
$$

where $U T$ - the set of user's tasks;

$U T_{i}-i$-th user's task;

$T_{i}$, - the title of $i$-th user's task;

$M D_{i p}-p$-th meta description of $i$-th user's task.

Scenarios set is described as:

$$
S c \ni S c_{k^{\prime}}
$$

where $S c$ - the set of scenarios;

$S c_{k}-k$-th scenario that is characterized by certain web services sequence.

Correlation between user's task and scenario (that corresponds to this user's task) is described by equivalence relation:

Web services set is described as:

$$
U T_{k} \sim S c_{k}
$$

$$
W S \ni W S_{l}, W S_{l}=\left\langle T_{l}, M D_{l q}\right\rangle
$$

where $W S$ - the set of web services;

$W S_{l}-l$-th web service;

$T_{b}$ - the title of $l$-th web service;

$M D_{l q}-q$-th meta description of $l$-th web service.

Correlation between the scenario and web services is described by inclusion relation (WSs are included into the scenario):

$$
S c_{k} \supset\left(W S_{l}, \ldots W S_{d}\right)
$$

It is in need to develop the method that enables scenario formation based on comparison of web services meta descriptions values and provides the ability to perform real end-user's tasks by forming sequence of web services dynamically. 


\section{The method of user's tasks scenario formation}

This section describes the steps of the proposed method of user's tasks scenario formation. This method allows to define dynamically an ordered sequence of web services required to execute a specific user's task. The stages of proposed method are represented below (Fig. 3).

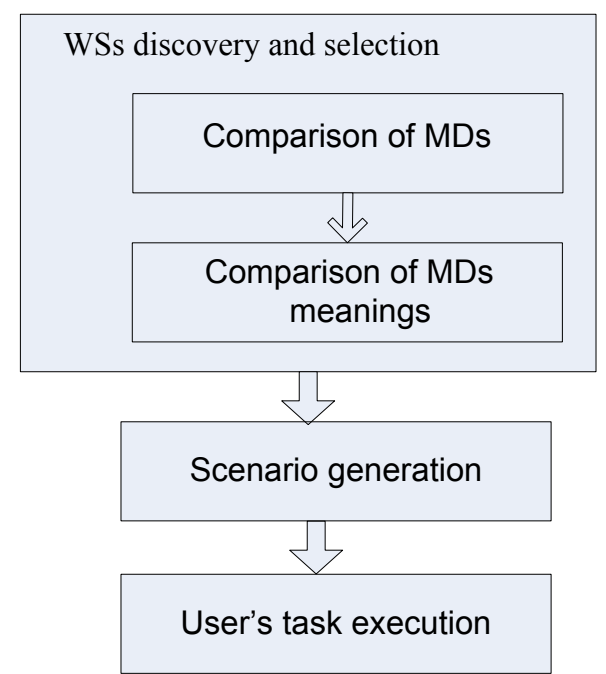

Fig. 3. Method of user's tasks scenario formation

Stage 1. WSs discovery and selection.

Stage 1.1. MD comparison.

On this stage it is in need to discard from the WSs set those WSs, for which all $M D_{l q}$ are not equal to $M D_{i v}$. It is in need to compare the descriptions of WSs and to select those WSs which meta descriptions match user's task meta descriptions according to the rule:

$$
M D_{i p}=\bigcup_{l}^{m} M D_{l q} .
$$

whereby possible the fulfilment: $M D_{l q} \cap M D_{d q}$, where $i, j \in l, l=\overline{1, m}$.

As the result the subset of WSs set is received, such subset satisfies rule (6).

Stage 1.2. MD meaning comparison.

It is necessary to compare meanings of web services meta description $M N\left(M D_{l q}\right)$ to cut the subset of web services, that has common meta descriptions, but the meanings of this meta descriptions are not equal. Thus, on this stage the values of WSs meta descriptions (only for those WSs that were left in the set after stage 1.1) are compared to the values of user's task meta description:

$$
M n\left(M D_{l q}\right)>M n\left(M D_{i v}\right),
$$

under the condition that $M D_{l q}=M D_{i v}$. The result can be one of the following options: 
1 Meta description value domains match $-M n\left(M D_{i v}\right)=M n\left(M D_{l q}\right)$. In this case, domain of the $v$-th meta description value of $i$-th user's task matches domain of $q$-th meta description value of $l$-th web service. Thus, specified web service is included in the user's task scenario.

2 One meta description value domain includes another one:

2.1. $M n\left(M D_{l q}\right) \subset M n\left(M D_{i v}\right)$. In this case, domain of the $q$-th meta description value of $l$-th web service includes domain of $v$-th meta description value of $i$-th user's task. Thus, specified web service is included in the user's task scenario.

2.2. $M n\left(M D_{i v}\right) \subset M n\left(M D_{l q}\right)$. In this case, domain of the v-th meta description value of $i$-th user's task includes domain of $q$-th meta description value of $l$-th web service. Thus, specified web service is included in the user's task scenario, but analysis of the meta description value for other web services continues in order to find such web service, which meta description value domain in combination with specified web service meta description value domain will form user's task meta description values domain: $M n\left(M D_{l q}\right) \cup M n\left(M D_{h d}\right)=\operatorname{Mn}\left(M D_{i v}\right)$.

3 Meta description value domains overlap - $\operatorname{Mn}\left(M D_{i v}\right) \cap M n\left(M D_{l q}\right)$. In this case, domain of the $v$-th meta description value of $i$-th user's task overlaps domain of $q$-th meta description value of $l$-th web service. Thus, specified web service is included in the user's task scenario, but analysis of the meta description value for other web services continues in order to find such web service, which meta description value domain in combination with specified web service meta description value domain will form user's task meta description values domain: $\operatorname{Mn}\left(M D_{l q}\right) \cup \operatorname{Mn}\left(M D_{h d}\right)=\operatorname{Mn}\left(M D_{i v}\right)$.

4 Meta description value domains don't overlap $-\operatorname{Mn}\left(M D_{l q}\right) \neq M n\left(M D_{i v}\right)$. In this case, domain of the $v$-th meta description value of $i$-th user's task matches domain of $q$-th meta description value of $l$-th web service. Thus, specified web service is not included in the user's task scenario.

Stage 2. Scenario generation.

This step can be regarded as an intermediate. At this stage, the sequence of the web services selected at the previous stage is formed into the scenario. Scenario formation involves redundancy and duplication verification. While the formation of WSs sequence is held it is in need to verify web services input and output data. If the result of any WS is the input data to another WS, then first WS should be placed before the second WS in the sequence.

Stage 3. User's task execution.

This stage is the stage of user's task direct execution. At this stage, the execution of all web services included into scenario is made using appropriate software.

\section{The estimation of the web services composition efficiency}

Suggested approach was used for scenarios that represents the user's tasks in subject domain "Strength of materials" formation [20,21]. To confirm the efficiency of the proposed method the test group of complex tasks for problem domain "Strength of materials" was selected (Tab. 2), which consists of five complex tasks. Each of 
complex tasks consists of several partial tasks (each partial tasks corresponds to certain web service).

Table 2

\begin{tabular}{|c|l|c|}
\hline № & \multicolumn{1}{|c|}{ The title of user's task } & $\begin{array}{c}\text { The amount of } \\
\text { stages (WSs) } \\
\text { in each user's } \\
\text { task }\end{array}$ \\
\hline 1 & $\begin{array}{l}\text { Calculation of the strength for the power components of } \\
\text { magnetic systems }\end{array}$ & 56 \\
\hline 2 & $\begin{array}{l}\text { Calculation of the strength for equipment and pipelines of } \\
\text { nuclear power systems }\end{array}$ & 28 \\
\hline 3 & ITER Structural Design Criteria for magnetic components & 33 \\
\hline 4 & Magnet DDD 1.1-1.3. Magnet System Design Criteria \\
\hline 5 & $\begin{array}{l}\text { Calculation of the strength for the elements of equipment } \\
\text { and pipelines of ship nuclear steam generating systems } \\
\text { with a water reactors }\end{array}$ & 18 \\
\hline
\end{tabular}

Let us show the example of complex task from the test group formation using proposed method. This task is «Calculation of the strength for the power components of magnetic systems». The system of meta description for such user's task is shown in table 3 .

Table 3

\begin{tabular}{|l|l|}
\hline Meta description title & Meta description value \\
\hline Title & $\begin{array}{l}\text { Calculation of the strength for the power components } \\
\text { of magnetic systems }\end{array}$ \\
\hline Identifier & 1 \\
\hline Subject field & Strength of materials \\
\hline Description & $\begin{array}{l}\text { The calculation, carried out for the cylinder formed by } \\
\text { the internal resistance of toroidal field coils }\end{array}$ \\
\hline Data & data, formulas \\
\hline Regulatory & PNAEG-7-002-86 \\
\hline Address & http $\backslash$ calc266\19.portal.ua \\
\hline Parameters & $\mathrm{a}_{1}=10-$ allowable pressure \\
& $\mathrm{E}_{2}=52-$ average loading \\
& $\mathrm{E}_{1}=48-$ basic loading \\
& $\mathrm{R}=5-$ winding radius \\
& $\mathrm{c}=10-$ the length of connection between \\
& construction body and winding \\
& $1=15-$ critical length \\
& $\mathrm{D}_{\mathrm{m}}=<10 \ldots 12>=$ maximal diameter \\
& $\mathrm{P}_{\mathrm{i}}=18-$ dimension of construction body \\
& $\eta=20-$ critical pressure \\
& $\phi=5-$ allowable tension pressure \\
& $\mathrm{v}=28-$ winding length \\
& $\mathrm{d}=44-$ winding strength \\
\hline
\end{tabular}




\begin{tabular}{|l|l|}
\hline & \multicolumn{1}{|c|}{$\begin{array}{c}\mathrm{M}=6-\text { constant of static level } \\
\mathrm{M}=12-\text { general membrane strength }\end{array}$} \\
\hline Loading & - \\
\hline Methods & - \\
\hline Element & Constructions shell, constructions corps \\
\hline Measurement area & Cross section, length \\
\hline
\end{tabular}

At the 1-st stage the analysis of WSs set that represents partial tasks was done. It was in need to choose from this set only those WSs that satisfies condition (6). Such WS are:

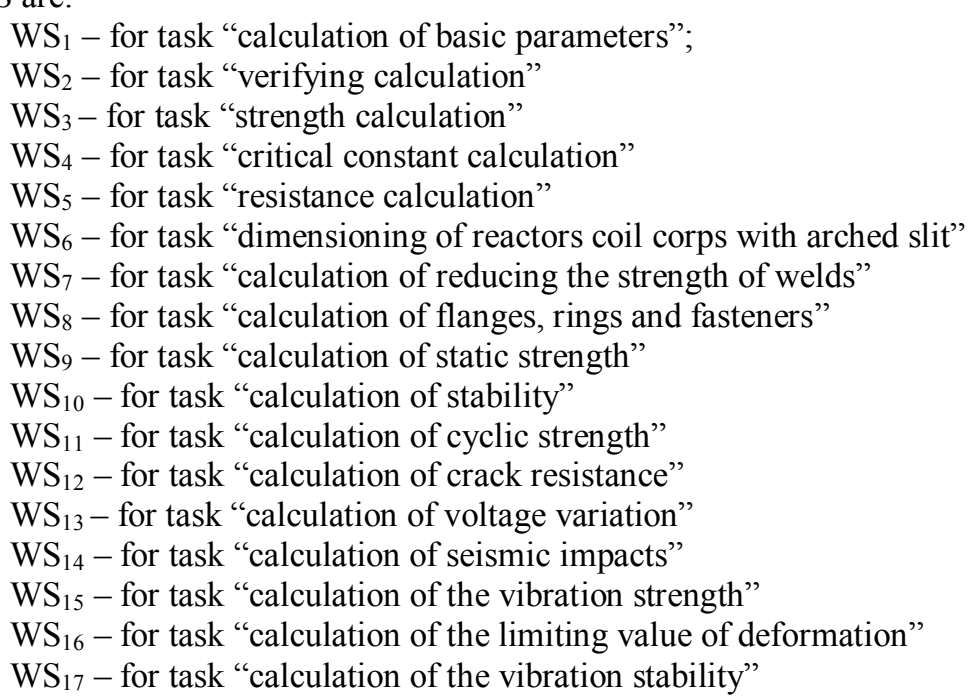

At 2-nd stage, the comparison of WSs meta descriptions meanings were held according to the rule (7). Some WSs were discarded from the set formed on the first stage, as some of their MD are out of range of values with values of the same parameters of task. The following WSs were discarded:

$\mathrm{WS}_{4}$ - for task "critical constant calculation"

$\mathrm{WS}_{10}$ - for task "calculation of stability"

$\mathrm{WS}_{11}$ - for task "calculation of cyclic strength"

$\mathrm{WS}_{13}$ - for task "calculation of voltage variation"

$\mathrm{WS}_{17}$ - for task "calculation of the vibration stability"

The estimation of the time required to develop scenarios for complex tasks was made. The estimation was made for static way of WSs composition and for their composition with the help of proposed method (Fig. 4). 


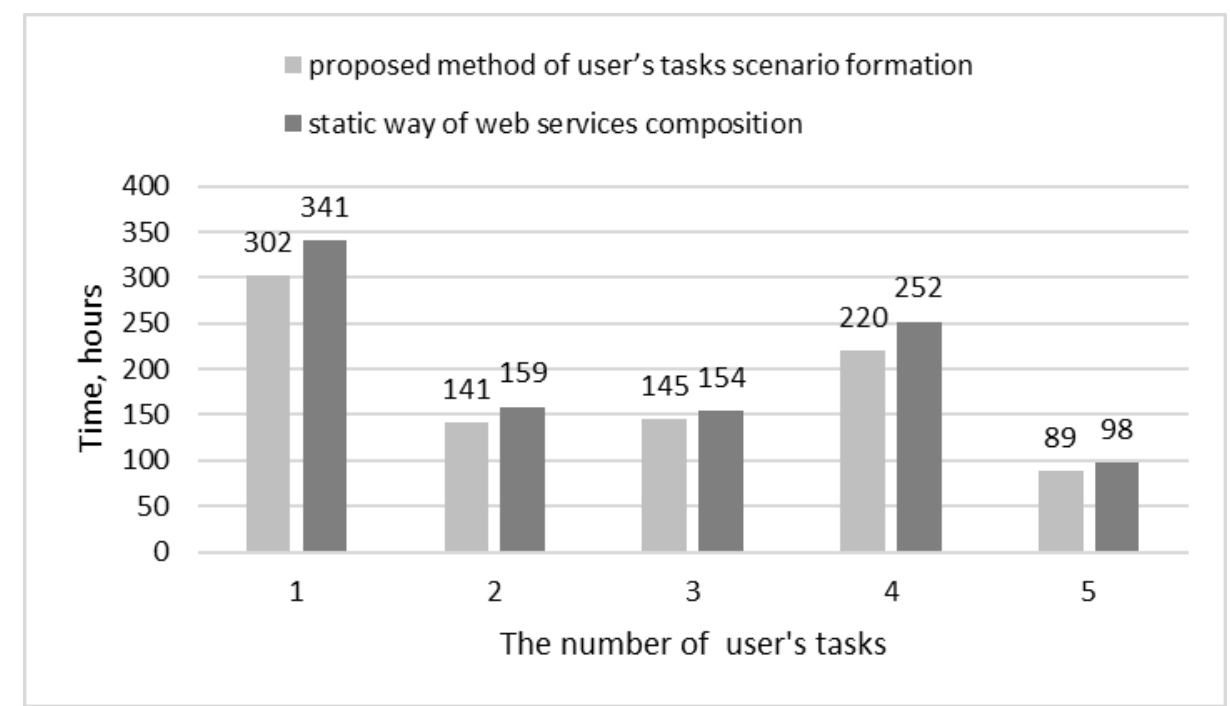

Fig. 4. Estimation of the WSs composition time

Time needed for services development with the help of static way WSs composition is 1004 hours and with the help of proposed method is 897 hours. Thus, the proposed method allowed to reduce the time for scenario for complex task development by $11 \%$.

\section{Conclusions}

The paper presents an approach to web services composition. The method of engineering tasks composition is proposed that allows to integrate web services into a scenario based on the web services meta descriptions comparison. The usage of such method makes it possible to execute real end-user's tasks based on dynamically formed sequence of web services.

Future work is aimed on implementation of suggested approach to different types of uses tasks. This will allow to approve its applicability and efficiency on real world scenarios. Developed software tool will be tested and verified on real world scenarios when engineering knowledge portals are developed. Quantitative evaluation of the proposed approach and tool efficiency will be obtained for different subject domains: the average time of web services composition, their correctness and quality will be validated.

\section{References}

1. Pukhkaiev, D., Kot, T., Globa, L., Schill, A.: A novel SLA-aware approach for web service composition. In: IEEE EUROCON, pp. 327--334 (2013) 
2. S. Dustdar, and W. Schreiner, "A survey on web services composition," in Int. J. Web and Grid Services, 2005, vol. 1, No. 1, p.1-30.

3. Sheng, Q. Z, Qiao, X., Vasilakos, A. V., Szabo, C., Boume, S., \& Xu, X. (2014). Web services composition: A decade's overview. Information Sciences 280, 218-238.

4. Moghaddam, M., \& Davis, J. G. (2014). Service selection in web service composition: A comparative review of existing approaches. In: Web Services Foundations (pp. 321-346). Springer New York.

5. Shehu, U., Epiphaniou, G., \& Safdar, G. A. (2014). A survey of QoS-aware web service composition techniques. International Journal of Computer Applications.

6. Upadhyaya, B. (2014). Composing Heterogeneous Services from End Users' Perspective.

7. Mcllraith, S. A., Son, T. C., \& Zeng, H. (2001). Semantic web services. IEEE intelligent systems, (2), 46-53.

8. Medjahed, B., \& Bouguettaya, A. (2011). Service composition for the Semantic Web. Springer Science \& Business Media.

9. Martin, D., Paolucci, M., McIlraith, S., Burstein, M., McDermott, D., McGuinness, D., ... \& Srinivasan, N. (2004). Bringing semantics to web services: The OWL-S approach. In Semantic Web Services and Web Process Composition (pp. 26-42). Springer Berlin Heidelberg.

10. Miller, J., Verma, K., Rajasekaran, P., Sheth, A., Aggarwal, R., \& Sivashanmugam, K. (2004). Wsdl-s: Adding semantics to wsdl-white paper.LSDIS Lab, University of Georgia, Georgia, USA.

11. De Oliveira Jr, F. G. A., \& de Oliveira, J. M. P. (2011). QoS-based Approach for Dynamic Web Service Composition. J. UCS, 17(5), 712-741.

12. Papazoglou, M. P., \& Van Den Heuvel, W. J. (2007). Service oriented architectures: approaches, technologies and research issues. The VLDB journal, 16(3), 389-415.

13. Hatzi, O., Vrakas, D., Nikolaidou, M., Bassiliades, N., Anagnostopoulos, D., \& Vlahavas, I. (2012).

14. An integrated approach to automated semantic web service composition through planning. Services Computing, IEEE Transactions on, 5(3), 319-332.

15. Ngan, L. D., \& Kanagasabai, R. (2013). Semantic Web service discovery: stateof-the-art and research challenges. Personal and ubiquitous computing, 17(8), 1741-1752

16. E.M. Maximilien and M.P. Singh., "A framework and ontology for dynamic web services selection,” IEEE Internet Computing, vol. 8, pp. 84-93, Sep./Oct. 2004.

17. M. B. Blake, and D. J. Cummings, "Workflow Composition of Service-Level Agreements," in Proceedings of IEEE International Conference on Services Computing (SCC), July 2007, p.138-145.

18. A. Andrieux, K. Czajkowski, A. Dan, K. Keahey, H. Ludwig, T. Nakata, J. Pruyne, J. Rofrano, S. Tuecke, and M. Xu. (2007) Web Services Agreement Specification (WSAgreement) Online]. Available:http://www.ogf.org/documents/GFD.107.pdf.

19. J. Cardoso, and A. Sheth, Semantic Web Services, Processes and Applications, Springer, 2006.

20. Norms for the strength calculation for the power components of magnetic systems. Kyiv: ISP, 1984. -73 c.

21. Norms for the strength calculation for equipment and pipelines of nuclear power systems - M.: Energoatomizdat, 1989. - 525 c. 